\title{
Risk factors for negative blood cultures in adult medical inpatients
} - a retrospective analysis

\author{
Boris P Ehrenstein*1, Vera Ehrenstein ${ }^{2}$, Christine Henke ${ }^{1}$, Hans-Jörg Linde ${ }^{3}$, \\ Bernd Salzberger ${ }^{1}$, Jürgen Schölmerich ${ }^{1}$ and Thomas Glück ${ }^{1}$
}

\begin{abstract}
Address: ${ }^{1}$ Dept. of Internal Medicine (I), University of Regensburg, Germany, ${ }^{2}$ Dept. of Epidemiology, School of Public Health, Boston University USA and ${ }^{3}$ Dept. of Medical Microbiology and Hygiene, University of Regensburg, Germany

Email: Boris P Ehrenstein* - boris.ehrenstein@klinik.uni-r.de; Vera Ehrenstein - vera.ehrenstein@gmail.com; Christine Henke - christinehenke@web.de; Hans-Jörg Linde - hans-joerg.linde@klinik.uni-r.de; Bernd Salzberger - bernd.salzberger@klinik.uni-r.de; Jürgen Schölmerich - juergen.schoelmerich@klinik.uni-r.de; Thomas Glück - thomas.glueck@klinik.uni-r.de

* Corresponding author
\end{abstract}

Published: 28 October 2008

BMC Infectious Diseases 2008, 8:148 doi:10.1 |86/147|-2334-8-148

Received: II October 2007

Accepted: 28 October 2008

This article is available from: http://www.biomedcentral.com//47| -2334/8/I48

(C) 2008 Ehrenstein et al; licensee BioMed Central Ltd.

This is an Open Access article distributed under the terms of the Creative Commons Attribution License (http://creativecommons.org/licenses/by/2.0), which permits unrestricted use, distribution, and reproduction in any medium, provided the original work is properly cited.

\begin{abstract}
Background: The identification of clinical factors associated with negative blood cultures could help to avoid unnecessary blood cultures. C-reactive protein (CRP) is a well-established inflammation marker commonly used in the management of medical inpatients.
\end{abstract}

Methods: We studied the association of clinical factors, CRP levels and changes of CRP documented prior to blood culture draws with the absence of bacteremia for hospitalized medical patients.

Results: In the retrospective analysis of 710 blood cultures obtained from 310 medical inpatients of non-intensive-care wards during one year (admission blood cultures obtained in the emergency room were excluded), the following retrospectively available factors were the only independent predictors of blood cultures negative for obligate pathogens: a good clinical condition represented by the lowest of three general nursing categories (OR 4.2, 95\% Cl I.8 - 9.5), a CRP rise > $50 \mathrm{mg} /$ $\mathrm{L}$ documented before the blood culture draw $(\mathrm{OR} 2.095 \% \mathrm{Cl}$ I.8-9.5) and any antibiotic treatment in the previous seven days (OR 2.0, $95 \% \mathrm{Cl}$ I.I-3.5).

Conclusion: Including the general clinical condition, antibiotic pre-treatment and a substantial rise of CRP into the decision, whether or not to obtain blood cultures from medical inpatients with a suspected infection, could improve the diagnostic yield.

\section{Background}

Blood cultures (BC) are especially important in the diagnosis of endovascular infections. At the same time, the benefits and cost effectiveness of blood culture diagnosis for many other infectious syndromes are less clearly defined. The identification of clinical factors associated with negative blood cultures could help to avoid unneces- sary BC. Serum C-reactive protein (CRP) is a well-established marker of inflammation $[1,2]$. It is commonly used in many clinical settings to evaluate and monitor severity of disease in patients with presumed infections [1]. We aimed to evaluate associations of different CRP levels or changes in CRP prior to BC draw with subsequent BC results. The association of negative $\mathrm{BC}$ results with other 
clinical factors commonly used to decide whether a BC draw is indicated and available by chart review were studied additionally.

\section{Methods}

At our tertiary care university hospital, we reviewed medical charts of all 363 patients from three general internal medicine non-intensive-care wards with at least one $\mathrm{BC}$ draw in the year 2000 (blood volume per BC app. 15 - 20 $\mathrm{mL}$, split and cultivated in an aerobic and an anaerobic bottle, BacT/Alert ${ }^{\mathrm{TM}}$, Organon Teknika, Durham, NC). During the study period, no formal guidelines for the indication of BC existed in our department. Ordering BC was therefore solely based on the judgement of the treating physicians.

Clinical relevance of skin flora isolated in BC (coagulasenegative staphylococci, Corrynebacterium spp., and Propionibacterium spp.) is difficult to determine, even prospectively. Therefore in our retrospective study, these bacterial species were regarded as either contaminations or the cause of endovascular catheter infections and were excluded from the analysis. All other isolated pathogens were considered to be obligate pathogens (OP).

We retrieved the following variables - all of which had been available to treating physicians at the time point of $\mathrm{BC}$-collection - and evaluated their association with $\mathrm{BC}$ results: $\mathrm{BC}$ draw in the emergency room (ER) or intensive care unit (ICU) in the previous 3 days; any antibiotic treatment in the previous 7 days; any intravenous antibiotics in previous 2 days; lack of high fever (temperature of < $40^{\circ} \mathrm{C}$ ); absence of documented substantial temperature rise (no rise or rise of $<2{ }^{\circ} \mathrm{C}$ ); absence of white blood cell count (WBC) elevation (value of $<12 / \mathrm{nL}$ ); absence of a documented substantial WBC increase (no rise or rise $<2$ / $\mathrm{nL}$ ); substantial (value of $>100 \mathrm{mg} / \mathrm{L}$ ) elevation of CRP level; documented substantial rise of CRP (rise of $>50$ $\mathrm{mg} / \mathrm{L}$ ); lowest general nursing category (reflecting dependence on help with daily activities, 1 vs. 2 or 3, as surrogate markers for the patients' overall clinical condition); age below 60 years. Only observations documented within 24 hours before the BC draw (within $48 \mathrm{~h}$ for WBC and CRP values) were included in the analysis. All factors reflecting changes over time were determined by the subtraction of the two most recent values obtained before the BC draw which were at least 24 hours apart. Additionally the main hospital discharge diagnosis of all patients with BC draws was obtained.

Crude analyses were performed using $\chi^{2}$-test, and adjusted analyses, using logistic regression, with the final predictive model derived by a backward elimination (exclusion criterion, $\mathrm{p}>0.05$ ). All analyses were performed using SAS software (SAS Institute Inc. Cary, NC).
The research was conducted in accordance with requirements set for retrospective studies by the local ethics committee (ethics committee of the University of Regensburg, Germany).

\section{Results}

A total of 856 BC from 363 patients on three analyzed non-ICU wards in the year 2000 were identified. 710 $(83 \%)$ of BC of $310(85 \%)$ patients had sufficient clinical documentation to be included in the analysis. 96 (13\%) of $710 \mathrm{BC}$ were positive, and in 91 the species could be identified. Thirty-two results were regarded as contamination or as related to endovascular catheter infection (coagulase-negative staphylococci $(\mathrm{n}=30)$, Corrynebacterium spp. $(\mathrm{n}=1)$, Propionibacterium acnes $(\mathrm{n}=1))$, and remaining 59 as obligate pathogens (OP): Gram-negative bacteria $\mathrm{n}=35$; Gram-positive bacteria $\mathrm{n}=16$; Candida spp. $\mathrm{n}$ $=8$ ). Comprising blood cultures drawn within 3 days as one diagnostic episode (DE), there were $426 \mathrm{DE}, 41$ $(10 \%)$ of which yielded OP. Of the 157 DE with more than $1 \mathrm{BC}, 17$ (11\%) DE yielded OP. An analysis stratified by the amount of $\mathrm{BC}$ per DE (1-2 vs. 3 or more $\mathrm{BC}$ per $\mathrm{DE})$ did not reveal a significant difference in the rate of OP-positive results $(35 / 445(7.9 \%)$ vs. $24 / 265(9.1 \%) \mathrm{p}=$ 0.57 by $\chi^{2}$-test). Furthermore, an analysis stratified by hospital day (BC obtained on day 1 and 2 vs. > day 2 of the hospital stay) showed no significant difference in the rate of OP-positve BC: $25 / 330$ (7.6\%) vs. $34 / 380$ (8.9\%); $\left(\mathrm{p}=0.51\right.$ by $\chi^{2}$-test $)$.

There were no significant differences ( $\chi^{2}$-test for 5 strata, $\mathrm{p}$ $=0.63)$ in the crude rates of OP-positive $\mathrm{BC}$ across strata of CRP values determined prior to drawing the BC: $0-5$ $\mathrm{mg} / \mathrm{L}$ (normal range) 2/20 (10.0\%), 6-50 mg/L 15/196 (7.7\%), 51-100 mg/L 14/133 (10.6\%), 101-200 mg/L $14 / 219(6.4 \%)$, and CRP > $200 \mathrm{mg} / \mathrm{L} 14 / 141$ (9.9\%). There was a significant difference $\left(\chi^{2}\right.$-test for 4 strata, $\mathrm{p}=$ 0.008 ) in the crude rates of OP-positive $\mathrm{BC}$ among strata of the documented change of CRP prior to drawing the BC: any fall in CRP 11/207 (5.3\%), CRP rise between 1 and $50 \mathrm{mg} / \mathrm{L} \mathrm{31/261}(11.9 \%)$, CRP rise > $50 \mathrm{mg} / \mathrm{L} 4 / 102$ $(3.9 \%)$, and change not applicable (only one CRP value determined prior to drawing the BC) $13 / 140$ (9.3\%). This difference remained statistically significant analyzing only the subgroup of $291 \mathrm{BC}$ from patients with no antibiotic therapy in the previous 7 days. For the subgroup of 61 BC from patients with any antibiotic therapy in the previous 7 days and a substantial rise of CRP (> $50 \mathrm{mg} / \mathrm{L}$ ) prior to drawing the $\mathrm{BC}$, the number of $\mathrm{BC}$ needed to diagnose one OP was $20(61 / 3)$.

A summary of crude and adjusted analyses is shown in Additional file 1 . In the crude analysis, the following factors were significantly associated with OP-negative BC: BC draw in the ER or ICU in the previous 3 days, absence of 
high fever, absence of a documented substantial temperature rise, absence of a documented substantial rise of the $\mathrm{WBC}$, and the lowest general nursing category. After considering all factors simultaneously in the logistic regression model (c-statistic 0.665; Hosmer-Lemeshow goodness-of-fit statistic 0.612 ; discordant prediction $20.4 \%$ ), low general nursing category (odds ratio (OR) 4.2 ), a CRP rise $>50 \mathrm{mg} / \mathrm{L}$ (OR 2.0), and any antibiotic in the previous 7 days (OR 2.0) remained significantly ( $<<$ 0.05 ) associated with OP-negative BC.

Analyzing the main hospital discharge diagnosis, 23/332 (6.9\%) BC drawn from patients with a non-infectious and $36 / 458$ (7.9\%) BC drawn from patients with an infectious main hospital discharge diagnosis were OP-positive. Among BC obtained from patients with infectious main hospital discharge diagnosis, OP-positive rates were as follows: abdominal infections 24/154 (15.6\%); soft tissue infections $1 / 58(1.7 \%)$; respiratory infections $1 / 86$ (1.2\%); sepsis $8 / 47$ (17.0\%); catheter infections $0 / 10$ $(0 \%)$; urogenital infections $0 / 9(0 \%)$; infections in HIVpositive patients $0 / 9(0 \%)$; endocarditis $2 / 2(100 \%)$; meningitis $0 / 3(0 \%)$.

\section{Discussion}

Several major studies have identified clinical factors and derived clinical prediction models for positive $\mathrm{BC}$ results [3-5]. These studies did not assess the role of CRP. We evaluated the association of CRP values and CRP change prior to obtaining $\mathrm{BC}$ with OP-negative $\mathrm{BC}$ results in adult internal medicine non-ICU patients and compared this association with that of selected other factors, commonly used in the decision on ordering $\mathrm{BC}$ available by chart review.

The level of CRP available before drawing the $\mathrm{BC}$ showed no association with $\mathrm{BC}$ results in our analysis. In accordance, a study of CRP values on the day of $\mathrm{BC}$ draw in patients with bacteremia showed no significant difference for CRP levels between OP-positive BC and contamination-positive $\mathrm{BC}$ [6]. Several studies, assessing the usefulness of CRP measured at the onset of fever in adult neutropenic cancer patients, showed no significant association $[7,8]$ or only a modest association with the presence of bacteremia [9]. In a retrospective analysis of adult inpatients with sepsis syndrome without shock, there was no significant difference of CRP values between bacteremic and non-bacteremic patients at the time of obtaining the BC [10]. Contrary to these studies, Tokuda et al. found in a population with a low prevalence $(4 \%)$ of malignant conditions a significant difference of CRP values between bacteremic and non-bacteremic ER patients with suspected infections [11]. They therefore included the variable of CRP $<100 \mathrm{mg} / \mathrm{L}$ in a classification algorithm for low risk of bacteremia.
There was an association between a substantial (> $50 \mathrm{mg} /$ $\mathrm{L}$ ) rise in CRP determined prior to BC draw and OP-negative $B C$ in this study. To our knowledge, so far no study has assessed changes of CRP prior to $\mathrm{BC}$ draw as a predictor for bacteremia among general internal medicine adult inpatients. At a first glance, it seems counterintuitive that a rise of an inflammation marker is associated with low prevalence of bacteremia. We speculate that this finding could be explained by the time-dependent course of CRP level after an inflammatory stimulus. Rintala and coworkers showed that CRP levels peak between 24 and 48 $h$ after admission for an infection $[2,12]$. Therefore, a substantial rise of CRP could indicate diagnostic situations, where the inflammatory stimulus - e.g. transient bacteremia - may already have disappeared by the time the $\mathrm{BC}$ is drawn. This hypothesis is supported by our finding of a low prevalence of OP-positive $\mathrm{BC}$ not only with a prior rise but also obtained after having passed a peak of CRP values prior to obtaining $\mathrm{BC}$.

A good overall clinical condition of the patient represented by the lowest of the 3 general nursing categories had the strongest association with OP-negative BC. We did not to evaluate disease-specific anamnestic information in our retrospective chart-based analysis. Therefore, the strong association of the overall clinical condition with $\mathrm{BC}$ results in the regression model was not surprising.

There are some limitations to our study. The retrospective design excluded the evaluation of certain anamnestic information and physical findings as competing clinical predictors. By design, we excluded BC from patients treated in the ICU. Therefore, our results might not apply to critical ill patients with a very high probability of bacteremia. The measurement of CRP was not performed at exactly specified time-points in relation to the obtaining of the BC. During the period studied, the measurement of procalcitonin as an inflammatory marker was clinically not available at our institution; therefore we could not evaluate the association of this promising new inflammatory marker with $\mathrm{BC}$ results in our retrospective cohort [13]. Because of the low frequency of positive $\mathrm{BC}$, we refrained from splitting our cohort in a derivation and a validation sample. Many (46.8\%) BC in our study were obtained from patients where the infection or presumed infection was not the main hospital discharge diagnosis. Therefore, our results should be confirmed in other clinical settings and especially the change of CRP prior to obtaining $\mathrm{BC}$ should be included in further prospective studies to learn more about the generalisability of our findings. 


\section{Conclusion}

The low rate of positive $\mathrm{BC}$ obtained from adult non-ICU inpatients with a passed or recent peak in CRP could be useful in the decision whether to draw or not to draw a blood culture. Recent changes in CRP or other inflammatory markers should be evaluated prospectively as candidate factors in future decision rules. Including the general clinical condition, antibiotic pre-treatment and a substantial rise of CRP into the decision, whether or not to obtain blood cultures from medical inpatients with a suspected infection, has the potential to improve the diagnostic yield of BC by refraining from BC-collection in situations with low positive-rates and therefore might lower costs.

\section{Competing interests}

The authors declare that they have no competing interests.

\section{Authors' contributions}

BPE designed the study, validated the chart review data, analyzed the data and wrote the manuscript, VE analyzed the data and revised the manuscript, $\mathrm{CH}$ performed the chart review and analyzed the data, HJL provided the microbiology data and revised the manuscript, BS, JS and TG designed the study and revised the manuscript.

\section{Additional material}

\section{Additional file 1}

Table 1 - Crude and adjusted analyses of the association of clinical factors with BC negative for obligate pathogens. A Microsoft Word document containing Table 1 of the manuscript.

Click here for file

[http://www.biomedcentral.com/content/supplementary/14712334-8-148-S1.doc]

\section{Acknowledgements}

This study was supported by research funds of the Department of Internal Medicine (I), University of Regensburg, Germany.

\section{References}

I. Povoa P: C-reactive protein: a valuable marker of sepsis. Intensive Care Med 2002, 28:235-243.

2. Gabay C, Kushner I: Acute-phase proteins and other systemic responses to inflammation. N Engl ] Med 1999, 340:448-454.

3. Bates DW, Cook EF, Goldman L, Lee TH: Predicting bacteremia in hospitalized patients. A prospectively validated model. Ann Intern Med 1990, I I 3:495-500.

4. Bates DW, Sands K, Miller E, Lanken PN, Hibberd PL, Graman PS, Schwartz JS, Kahn K, Snydman DR, Parsonnet J, et al.: Predicting bacteremia in patients with sepsis syndrome. Academic Medical Center Consortium Sepsis Project Working Group. J Infect Dis 1997, I 76: 1538-I55I.

5. Leibovici L, Greenshtain S, Cohen O, Mor F, Wysenbeek AJ: Bacteremia in febrile patients. A clinical model for diagnosis. Arch Intern Med I99I, 15 I:I80I-I806.

6. McCabe RE, Remington JS: C-reactive protein in patients with bacteremia. J Clin Microbiol 1984, 20:3 17-319.

7. de Bont ES, Vellenga E, Swaanenburg JC, Fidler V, Visser-van Brummen PJ, Kamps WA: Plasma IL-8 and IL-6 levels can be used to define a group with low risk of septicaemia among cancer patients with fever and neutropenia. Br J Haematol 1999, 107:375-380.

8. Engel A, Mack E, Kern P, Kern WV: An analysis of interleukin-8, interleukin-6 and C-reactive protein serum concentrations to predict fever, gram-negative bacteremia and complicated infection in neutropenic cancer patients. Infection 1998, 26:2|3-22|.

9. Persson L, Engervall P, Magnuson A, Vikerfors T, Soderquist B, Hansson LO, Tidefelt U: Use of inflammatory markers for early detection of bacteraemia in patients with febrile neutropenia. Scand J Infect Dis 2004, 36:365-37I.

10. Byl B, Deviere J, Saint-Hubert F, Zech F, Gulbis B, Thys JP: Evaluation of tumor necrosis factor-alpha, interleukin-6 and $C$ reactive protein plasma levels as predictors of bacteremia in patients presenting signs of sepsis without shock. Clin Microbiol Infect 1997, 3:306-316.

II. Tokuda $\mathrm{Y}$, Miyasato $\mathrm{H}$, Stein $\mathrm{GH}$ : A simple prediction algorithm for bacteraemia in patients with acute febrile illness. QJM 2005, 98:813-820.

12. Rintala EM, Aittoniemi J, Laine S, Nevalainen TJ, Nikoskelainen J: Early identification of bacteremia by biochemical markers of systemic inflammation. Scand J Clin Lab Invest 2001, 6 1:523-530.

13. Jones AE, Fiechtl JF, Brown MD, Ballew JJ, Kline JA: Procalcitonin Test in the Diagnosis of Bacteremia: A Meta-analysis. Ann Emerg Med 2007, 50:34-4I.

\section{Pre-publication history}

The pre-publication history for this paper can be accessed here:

http://www.biomedcentral.com/1471-2334/8/148/pre pub

Publish with BioMed Central and every scientist can read your work free of charge

"BioMed Central will be the most significant development for disseminating the results of biomedical research in our lifetime. " Sir Paul Nurse, Cancer Research UK

Your research papers will be:

- available free of charge to the entire biomedical community

- peer reviewed and published immediately upon acceptance

- cited in PubMed and archived on PubMed Central

- yours - you keep the copyright 